\title{
Factores asociados a sobrevida en pacientes con co-infección VIH-TBC en el Servicio de Infectología del Hospital Nacional Arzobispo Loayza, Perú, durante los años 2004-2012
}

\author{
Brenda Gamboa-Acuña, Rayza Guillén-Zambrano, Grecia Lizzetti-Mendoza, \\ Alonso Soto, Aldo Lucchetti-Rodríguez
}

Factors associated to survival in patients with HIV-TB in the Department of Infectious Diseases of the Arzobispo Loayza National Hospital, Perú, since 2004 to 2012

Background: The main cause of death in HIV patients is tuberculosis (TB). However, few Latin American studies have evaluated the prognosis of patients with coinfection. Aim: To determine the factors associated with survival in patients with HIV-TB coinfection treated at a Peruvian referral hospital. Methods: A retrospective cohort study was performed based on clinical records of patients treated at the Department of Infectious Diseases in the Arzobispo Loayza National Hospital from 2004 to 2012. Survival was assessed using the Kaplan-Meier estimator and Cox Proportional Hazard Model. Results: From 315 patients, 82 died during the follow-up. The mean of follow for each patient was 730 days. The multivariate analysis showed that receiving HAART (HR: 0,31; IC: 0,20-0,50; $\mathrm{p}<0,01$ ) and having more weight (HR: 0,96; IC 0,94-0,98; $<<0,01$ ) when the coinfection was diagnosed, were protective factors; while having a pathology different from TB (HR: 1,88; IC: 1,19-2,98; $\mathrm{p}<0,01$ ), age in years (HR: 1,76; IC: 1,12-2,74; $\mathrm{p} \leq 0,01$ ) and being hospitalized when diagnosed with TB (HR: 1,69; IC 1,02-2,80; $\mathrm{p}<0,04)$ were associated with lower survival. Discussion: Receiving HAART and having more weight when the coinfection is diagnosed were associated with a higher chance of survival.

Key words: Tuberculosis, HIV, survival, mortality

Palabras clave: Tuberculosis, VIH, sobrevida, mortalidad.

\section{Introducción}

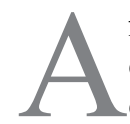

finales del año 2016, 36,7 millones de personas en el mundo vivían infectadas por el $\mathrm{VIH}^{1}$. Desde el comienzo de la epidemia, alrededor de 78 millones de personas contrajeron la infección por VIH, y 39 millones han fallecido a causa de enfermedades relacionadas con este virus ${ }^{2}$. En el 2012, América Latina concentró un promedio de 1,5 millones de casos, mientras que, en el 2015, en Perú, se reportaron 1.235 casos de infección por VIH y 5.257 de SIDA. Lima Metropolitana continúa siendo la ciudad con mayor reporte de casos de infección por $\mathrm{VIH}$, con una incidencia acumulada desde el año 2004 al año 2015 de 20.778 pacientes con VIH y 10.077 con SIDA $^{1,3}$.

La principal causa de muerte en este grupo de pacientes es la tuberculosis (TBC), especialmente en aquellos que viven en países de ingresos bajos a moderados, incluyendo a quienes reciben terapia anti-retroviral de gran actividad $\left(\right.$ TARGA ${ }^{4-6}$. En comparación con pacientes libres de infección por VIH, los pacientes seropositivos tienen una probabilidad hasta 50 veces mayor de desarrollar TBC a lo largo de su vida ${ }^{7}$. En el año 2015, la Organización
Mundial de la Salud (OMS) reportó 1,2 millones (11\%) de casos nuevos de co-infección VIH-TBC ${ }^{8}$. La presentación de TBC en Perú, como infección oportunista en pacientes con infección por VIH es de $28 \%$ y de hasta $50 \%$ en pacientes en estadio SIDA $^{9}$. Sin TARGA, cerca de $90 \%$ de seropositivos fallece dos a tres meses después de haber contraído la TBC, y aún con tratamiento anti-retroviral, los pacientes con TBC fallecen antes que aquellos libres de infección ${ }^{10}$.

La co-infección VIH-TBC altera la presentación clínica habitual de ambas enfermedades, dificultando el diagnóstico y manejo oportuno. En este grupo de pacientes, el diagnóstico de TBC representa un reto para el médico clínico debido a la frecuente presentación asintomática, patrones radiológicos atípicos y negatividad del examen directo de esputo ${ }^{11}$. La presentación clínica y radiológica de la TBC en pacientes con infección VIHTBC está relacionada con sus recuentos de linfocitos T (LT) CD4+, ya que con recuentos mayores de 350 céls/ $\mathrm{mm}^{3}$ la presentación es igual que en personas que no tienen infección por VIH. Por otro lado, si el recuento es menor, la radiografía suele ser atípica con afección de cualquier parte del parénquima pulmonar ${ }^{12,13}$. Además, las
Escuela de Medicina. Universidad Peruana de Ciencias Aplicadas, Lima-Perú (BG-A, RG-Z, GL-M, AS). Hospital Nacional Hipólito Unánue. Lima, Perú (AS). Hospital Nacional Arzobispo Loayza. Lima, Perú (AL-R).

Los autores declaran no tener conflictos de interés.

Recibido: 27 de febrero de 2017 Aceptado: 15 de enero de 2018

Correspondencia a: Rayza Elizabeth Guillén Zambrano raeguiza@gmail.com 
personas con infección por VIH tienen mayor riesgo de una reactivación de la TBC latente y contraer una nueva infección, en relación a personas no infectadas por $\mathrm{VIH}^{13}$.

Se ha demostrado que el esquema TARGA restaura considerablemente la inmunidad y reduce la morbimortalidad en pacientes infectados por VIH, disminuyendo así el riesgo de adquirir $\mathrm{TBC}^{14}$. Incluso después de adquirir la infección tuberculosa, el tratamiento se asocia a mejores tasas de supervivencia ${ }^{15}$.

En febrero de 2004, en el Perú, se aprobó la Norma Técnica de TARGA en Adultos y marcó el hito del principio de la era del TARGA a nivel nacional. Tres meses después, el Ministerio de Salud, en colaboración con el Fondo Global de lucha contra el SIDA, la tuberculosis y la malaria implementó el Programa a fin de brindar tratamiento en forma gratuita. Se inició el suministro en tres hospitales limeños, siendo el Hospital Nacional Arzobispo Loayza uno de los pioneros en su distribución ${ }^{16,17}$. Sin embargo, los datos en relación a la sobrevida en pacientes con co-infección VIH-TBC son escasos. Un estudio realizado en 1994 previo a la disponibilidad de TARGA en el sistema de salud peruano, encontró que los pacientes con dicha co-infección tenían un tiempo promedio de sobrevida de 7,2 meses a diferencia de aquellos que sólo tenían la infección por VIH, cuya sobrevida fue de 10,4 meses $^{18}$.

Existen estudios que han evaluado los factores asociados a mortalidad en pacientes con co-infección VIH-TBC. Entre los factores más consistentemente asociados se encuentran tener un peso menor de $50 \mathrm{~kg}$, la duración

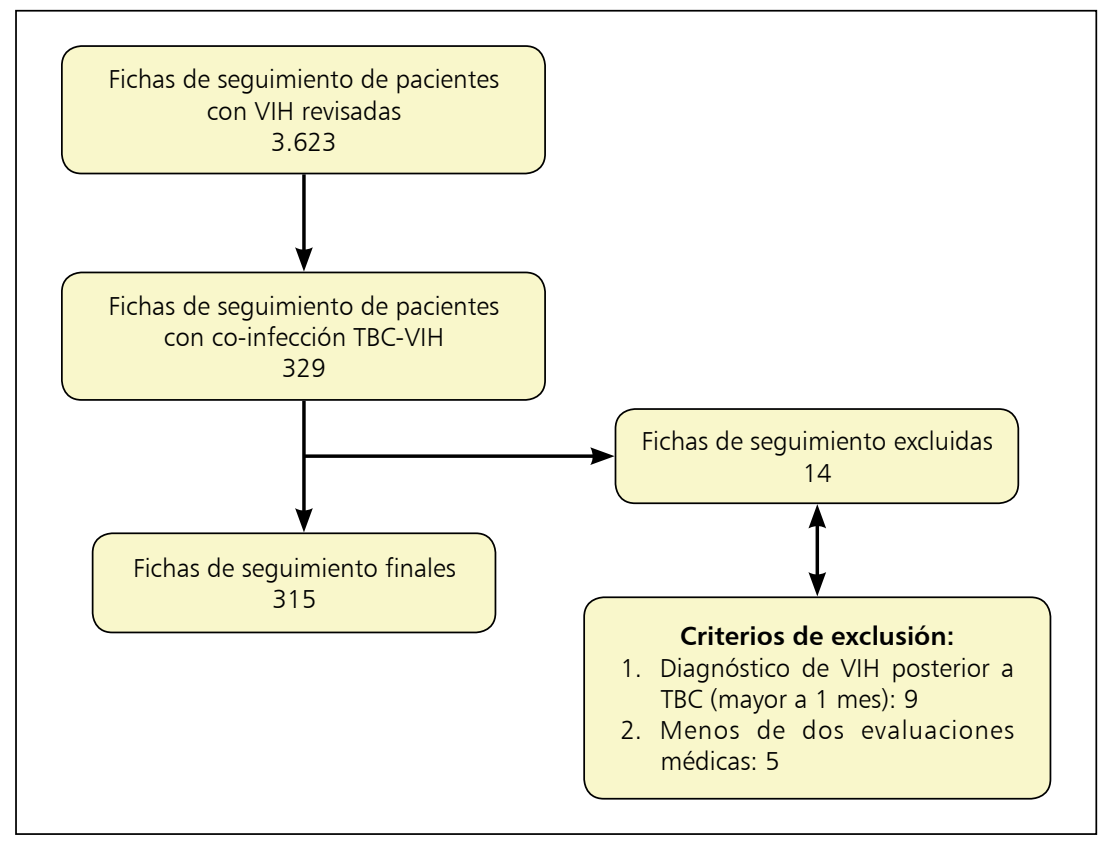

Figura 1. Selección de la población de estudio. Hospital Nacional Arzobispo Loayza, Lima-Perú. 2004-2012. de la infección por VIH por más de 12 meses; presencia de otras enfermedades oportunistas, co-morbilidades crónicas no relacionadas al SIDA, no recibir profilaxis con cotrimoxazol ni tratamiento con TARGA y tener un recuento de LT CD4 menor a 200 céls $/ \mathrm{mm}^{3}$ son factores asociados con mortalidad ${ }^{19}$. A pesar del gran esfuerzo realizado por disminuir el impacto de la co-infección VIH$\mathrm{TBC}$, aún existen factores que dificultan la sobrevida en esta población. En el Perú no existen estudios que hayan evaluado factores predictores del pronóstico en este grupo de pacientes en la era TARGA.

El objetivo del estudio fue determinar los factores asociados a sobrevida en pacientes con co-infección VIHTBC atendidos en el Servicio de Infectología de un hospital peruano de referencia, durante los años 2004-2012.

\section{Materiales y Métodos}

\section{Diseño y lugar de estudio}

Se elaboró un estudio de tipo cohorte, retrospectivo, en base a fichas de seguimiento de pacientes con diagnóstico de co-infección por el VIH y TBC atendidos durante los años 2004 al 2012 en el Servicio de Infectología del Hospital Nacional Arzobispo Loayza en Lima, Perú.

\section{Población}

Se incluyeron a todos los pacientes atendidos durante el período de estudio con información disponible en las fichas de seguimiento, pertenecientes a alguno de los tres escenarios: pacientes con antecedente de infección por VIH a quienes se les diagnosticara TBC, pacientes a quienes se les diagnosticó VIH y TBC simultáneamente y pacientes con TBC a quienes se les diagnosticara VIH hasta un mes después. Los pacientes que no se encontraban en estos tres escenarios o que tuvieran menos de dos evaluaciones médicas desde el diagnóstico de la co-infección (necesarias para tener un mínimo de información confiable a fin de realizar el análisis) fueron excluidos del estudio (Figura 1).

\section{Procedimientos}

El estudio fue aprobado por el Comité de Ética de la Universidad Peruana de Ciencias Aplicadas y la Oficina de Apoyo a la Docencia e Investigación del Hospital Nacional Arzobispo Loayza. Se conservó la privacidad y confidencialidad de las fichas de seguimiento y/o historias clínicas.

Los datos obtenidos a partir de las fichas de seguimiento fueron ingresados a una base de datos elaborada utilizando Microsoft Access mediante doble digitación para asegurar el control de calidad. Para las variables cuantitativas con distribución normal, se calcularon la media y desviación estándar, mientras que para las de distribución no normal se optó por mediana y rango intercuartil. Las variables categóricas se presentaron como 
frecuencias y porcentajes. La función de sobrevida global fue graficada mediante curvas de Kaplan Meier, el análisis crudo de factores asociados a la sobrevida de los pacientes con co-infección VIH-TBC fue realizado mediante el método de Log Rank Test y el análisis ajustado mediante el método de Riesgos Proporcionales de Cox. Se elaboró un primer modelo multivariado utilizando todas aquellas variables con un valor de $\mathrm{p}<$ de 0,15 en el análisis bivariado. A continuación, se eliminaron en forma sucesiva aquellas variables con mayores valores de $\mathrm{p}$ (backward elimination) hasta lograr un modelo ajustado final en el que todas las variables presentaran un valor de $\mathrm{p}$ menor a 0,05. Para el análisis se utilizó el paquete estadístico Stata versión 12 para Windows.

\section{Cálculo del tamaño muestral}

Se incluyeron a todos los pacientes atendidos durante el período de estudio; sin embargo, para fines referenciales se realizó el cálculo del tamaño muestral utilizando el comando stpower (Stata 11). Considerando una potencia estadística de $80 \%$ y un HR de 1,5 como clínicamente relevante, el tamaño muestral calculado fue de 198 participantes $^{19,20}$.

\section{Definición de variables}

Al ser un estudio de sobrevida, el resultado primario fue el tiempo transcurrido hasta el desarrollo del evento, definiéndose este último como muerte por cualquier causa. Se incluyeron como variables independientes: edad en años, género (femenino, masculino), lugar de procedencia (Lima, provincia), grado de instrucción (ninguno, primaria, secundaria, técnica, superior), riesgo de depresión (evaluado mediante entrevista psicológica y test de Machover), hospitalización (Sí/No), tipo de TBC (pulmonar o extrapulmonar), confirmación bacteriológica (baciloscopia, cultivo, prueba molecular y no disponible) patología distinta a TBC (patología crónica no infecciosa, patología infecciosa), esquema TARGA (sin TARGA, esquema basado en efavirenz, nevirapina o en inhibidor de la proteasa reforzado con ritonavir), fecha de diagnóstico de infección por VIH, fecha de diagnóstico de TBC, fecha de inicio de TARGA, año de constatación de la co-infección, alanina aminotransferasa $(\leq 40 \mathrm{U} / \mathrm{L}$ $\mathrm{O}>40 \mathrm{U} / \mathrm{L})$, hemoglobina $(<12 \mathrm{~g} / \mathrm{dL} \mathrm{o} \geq 12 \mathrm{~g} / \mathrm{dL}$ en mujeres $\mathrm{y}<13 \mathrm{~g} / \mathrm{dL} \mathrm{o} \geq 13 \mathrm{~g} / \mathrm{dL}$ en varones), recuento de $\mathrm{LT}$ CD4+ $\left(<200\right.$ céls $/ \mathrm{mm}^{3} \mathrm{y} \geq 200$ céls $\left./ \mathrm{mm}^{3}\right)$, carga viral en logaritmo base 10, linfadenopatías (Sí/No), peso en $\mathrm{kg}$, radiografía de tórax (infiltrados apical, cavitario o miliar), correspondiendo todas estas variables al momento del diagnóstico de TBC. La variable dependiente fue condición del paciente al término del estudio.

Para el caso de los pacientes con TBC sin comprobación microbiológica ni radiológica, se consideraron positivos a aquellos que cumplieron los criterios clínicos establecidos por la Norma Técnica de Salud para la Atención Integral del Adulto con infección por el Virus de la Inmunodeficiencia Humana (VIH).

\section{Tiempo de sobrevida}

Se consideró como el tiempo en días que transcurrió entre el diagnóstico de la co-infección y el fallecimiento del paciente, o en su defecto, 730 días desde el diagnóstico de la co-infección. Los casos vivos al cierre del estudio, se consideraron censurados.

\section{Análisis estadístico}

Para el análisis bivariado se empleó la prueba estadística Log Rank Test, para lo cual fue necesario categorizar las variables numéricas (edad, peso y carga viral al momento del diagnóstico de la co-infección) por encima y por debajo de la mediana. Todas aquellas variables con un valor de $\mathrm{p}<0,05$ fueron consideradas como significativas. Sin embargo, para efectos del análisis multivariado con Regresión de Cox, se incluyeron a aquellas con un $\mathrm{p}<0,15$. En este, las variables numéricas fueron utilizadas como tal, a excepción de edad. Luego, mediante el método de backward elimination, se seleccionaron las variables que finalmente mantenían un $\mathrm{p}<0,05$, considerándose estas significativas con la variable de respuesta. Los datos se procesaron y analizaron con el software Stata 12.

\section{Resultados}

\section{Descripción de la población}

Se revisaron 3.623 registros de pacientes con infección por VIH atendidos en el Servicio de Infectología del hospital de estudio. De éstos, se seleccionaron 329 correspondientes a los pacientes con co-infección VIHTBC entre los años 2004 a 2012; sin embargo, 14 fueron excluidos por no cumplir con los criterios de inclusión, quedando un total de 315 registros para el análisis final del estudio (Figura 1). El año 2008 presentó la mayor cantidad de pacientes con co-infección VIH-TBC, con un total de 42 pacientes. Del total de sujetos de estudios, $83(26,3 \%)$ fallecieron.

La mediana de edad fue de 34 años, siendo 155 (49,2\%) pacientes mayores a esta edad; mientras que $246(78,1 \%)$ pacientes fueron de género masculino. Del total de pacientes, 52 (16,5\%) estuvieron hospitalizados al momento del diagnóstico de la co-infección. Efavirenz fue el núcleo de tratamiento TARGA en $38,1 \%$ de los pacientes.

La presentación más frecuente de TBC en la población fue de tipo pulmonar (62,2\%). En $82 \%$ de los casos el diagnóstico se realizó mediante baciloscopia. Sólo $3,1 \%$ fue diagnosticado mediante cultivo. El 37,8\% de radiografías de tórax fueron informadas como normales, mientras que $22,9 \%$ presentó un patrón no sugerente de 


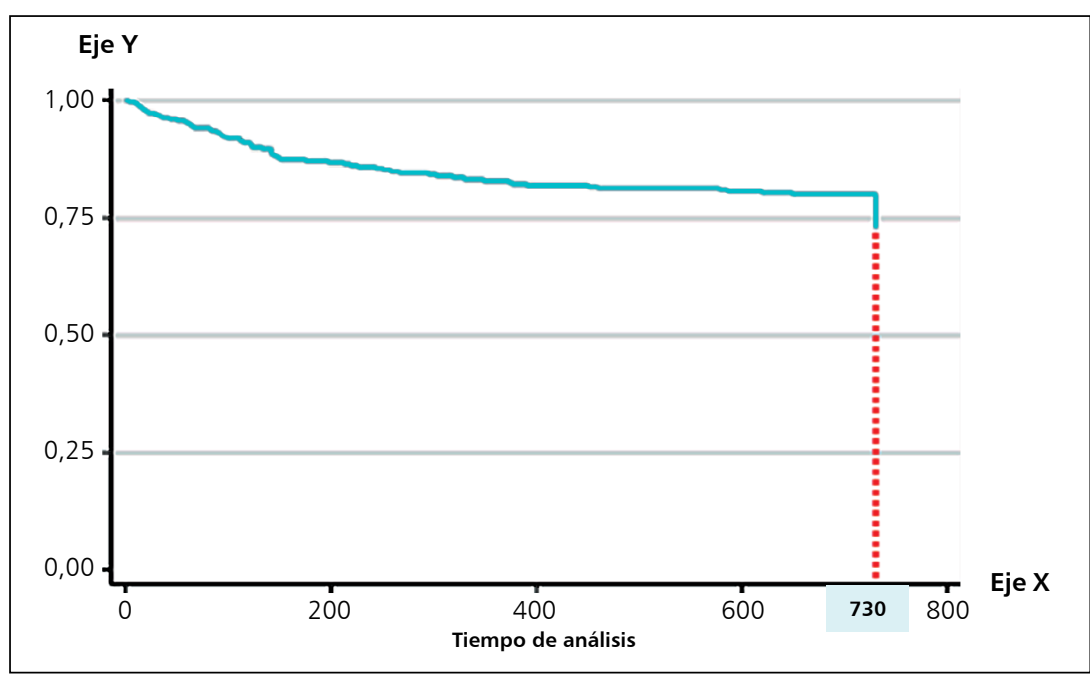

Figura 2. Curva de Kaplan Meier, sobrevida a dos años de la población de estudio. Hospital Nacional Arzobispo Loayza, Lima-Perú. 2004-2012. Eje X: Tiempo de seguimiento en días. Eje Y: Proporción de pacientes libres del evento.
TBC. El 11,4\% de los pacientes presentó TBC multiresistente.

La principal patología infecciosa oportunista concomitante fue la infección por Pneumocystis jiroveci (4,4\%), mientras que la infección por Treponema pallidum, fue la patología infecciosa no oportunista con mayor prevalencia (7,3\%). El 29,2\% de los pacientes evidenció riesgo de depresión en la evaluación psicológica previa al ingreso al programa TARGA. Del total de pacientes, $21,9 \%$ presentaron anemia al momento del diagnóstico de la co-infección; de estos, 51 (16,2\%) fueron mujeres y $18(5,7 \%)$ fueron varones. Finalmente, la mediana de carga viral fue de $11,74 \log _{10}$ copias de ARN/ml y 195 $(61,9 \%)$ pacientes presentaron un valor de LT CD4+ menor a 200 céls $/ \mathrm{mm}^{3}$.

\section{Sobrevida y factores asociados}

La mediana de seguimiento fue de 730 días. De acuerdo a la curva de Kaplan Meier, la mayor caída en la probabilidad de sobrevida ocurrió antes de los 200 días, luego de los cuales la curva desciende lentamente hasta tornarse estacionaria (Figura 2). Se presentaron 83 muertes representando una tasa de mortalidad de 15,9 muertes/100 personas año de seguimiento. En el análisis bivariado, la edad, hospitalización, peso, esquema de TBC, esquema de TARGA y patología diferente a TBC al momento del diagnóstico de la co-infección, se asociaron significativamente a una menor sobrevida. (Tablas 1 a 4). En el análisis multivariado basado en el método de riesgos proporcionales de Cox, y tras emplear la técnica de backward elimination, se observó que las variables peso (HR: 0,96; IC 0,94-0,98; p < 0,01) y esquema de TARGA (HR 0,31; IC: 0,20-0,50; $<<0,01$ ) fueron factores protectores; mientras que edad (HR 1,76; IC: 1,12-2,74; $p<0,01$ ), estar hospitalizado (HR: 1,69; IC: 1,02-2,80; $<<0,04)$, y patología diferente a TBC (HR: 1,88; IC: 1,19-2,98; $\mathrm{p}<0,01)$ se asociaron a una menor sobrevida. (Tabla 5) La adición del recuento de LT CD4 y carga viral al modelo multivariado final no modificaron los resultados obtenidos.

\section{Discusión}

La co-infección VIH-TBC continúa siendo una importante causa de mortalidad en escenarios de recursos limitados. Por ello es importante identificar aquellos factores asociados al pronóstico de esta condición a fin de identificar a los pacientes con mayor vulnerabilidad e intervenir sobre aquellos factores modificables. Este estudio evidenció que la edad, el estar hospitalizado al momento del diagnóstico de la co-infección y cursar con una patología infecciosa diferente a TBC, fueron los factores asociados a una menor sobrevida. Por otro lado, 
Tabla 2. Tipo de tuberculosis y confirmación bacteriológica en pacientes con co-infección VIH-TBC y su asociación con sobrevida. Lima, Perú. 2004-2012

\begin{tabular}{|c|c|c|}
\hline Variable & Total $(n=315) \%$ & Valor $\mathrm{p}^{*}$ \\
\hline Año de coinfección & & 0,67 \\
\hline $2004-2006$ & $91(28,9)$ & \\
\hline 2007-2009 & $119(37,8)$ & \\
\hline $2010-2012$ & $105(33,5)$ & \\
\hline Tipo de tuberculosis & & 0,09 \\
\hline Pulmonar & $196(62,2)$ & \\
\hline Ganglionar & $30(9,6)$ & \\
\hline Pleural & $21(6,7)$ & \\
\hline Meníngea & $23(7,3)$ & \\
\hline Otras & $45(14,3)$ & \\
\hline Confirmación bacteriológica & & 0,27 \\
\hline Baciloscopia & $258(82)$ & \\
\hline Cultivo & $10(3,1)$ & \\
\hline Prueba molecular & $17 \quad(5,4)$ & \\
\hline No disponible & $20(6,4)$ & \\
\hline Sin confirmación bacteriológica & $10(3,2)$ & \\
\hline Esquema de tuberculosis & & 0,03 \\
\hline Sensible & $279(88,8)$ & \\
\hline Multi-resistente & $36(11,4)$ & \\
\hline
\end{tabular}

*Basado en Log Rank Test. Las variables categóricas, se muestran como frecuencias y porcentajes.

el recibir TARGA y tener un mayor peso al momento del diagnóstico de la co-infección se asociaron a un menor riesgo de mortalidad.

Según el área geográfica, la TBC es responsable de la muerte de 11 hasta $50 \%$ de pacientes con infección por $\mathrm{VIH}^{21}$. El hospital de referencia en el cual se realizó el presente estudio contaba con una población total de 3.623 pacientes con infección por $\mathrm{VIH}$, atendidos entre los años 2004-2012. De ellos, 12\% presentó además, infección por Mycobacterium tuberculosis, siendo ésta nuestra población de estudio.

La incidencia de muerte obtenida fue de 15,9 muertes/100 personas/ año de seguimiento, a diferencia de un estudio realizado por Catalá y cols., cuya incidencia de muerte fue $8,6 / 100$ personas/año, durante 10 años de seguimiento ${ }^{22}$.

La edad ha mostrado ser un predictor importante de mortalidad en varios estudios incrementando el riesgo hasta en $28 \%$, sobre todo en los pacientes mayores de 50 años ${ }^{23}$. En base a los resultados obtenidos, se encontró que la mediana de edad en pacientes con co-infección VIH-TBC fue de 34 años, con un rango intercuartil de 29-43 años, dato que se aproxima a los reportados en estudios realizados en Malasia y Camerún donde la mediana fue de 39,1 y 39,3 años; respectivamente ${ }^{19,20}$. El presente estudio mostró que los pacientes co-infectados que sean mayores de 34 años presentan un riesgo de mortalidad de $76 \%$ por cada año de vida obtenido.

Estar hospitalizado se asocia generalmente a un mayor compromiso del estado general del paciente, lo cual empeora su pronóstico. En diferentes unidades de internación del Departamento de Infectología
Tabla 3. Co-morbilidades y su asociación con sobrevida en pacientes con co-infección VIH-TBC. Lima, Perú. 2004-2012

\begin{tabular}{|c|c|c|}
\hline Variable & Total $(n=315)-\%$ & Valor $\mathrm{p}^{*}$ \\
\hline $\begin{array}{l}\text { Patología diferente a tuberculosis pulmonar } \\
\text { Sí }\end{array}$ & $86(27,3)$ & 0,02 \\
\hline $\begin{array}{l}\text { Patología oportunista } \\
\text { Ninguna } \\
\text { Pneumocystis jiroveci } \\
\text { Cryptococcus neoformans } \\
\text { Toxoplasma gondii } \\
\text { Otras }\end{array}$ & $\begin{array}{rr}282 & (89,6) \\
14 & (4,4) \\
9 & (2,9) \\
9 & (2,9) \\
1 & (0,3)\end{array}$ & 0,07 \\
\hline $\begin{array}{l}\text { Patología no oportunista } \\
\text { Ninguna } \\
\text { Hepatitis B } \\
\text { Hepatitis C } \\
\text { Sífilis } \\
\text { Otras }\end{array}$ & $\begin{array}{rr}269 & (85,4) \\
12 & (3,8) \\
10 & (3,2) \\
23 & (7,3) \\
1 & (0,3)\end{array}$ & 0,40 \\
\hline $\begin{array}{l}\text { Patología no infecciosa } \\
\text { Diabetes mellitus } \\
\text { Hipertensión arterial } \\
\text { Dislipidemia } \\
\text { Otras }\end{array}$ & $\begin{array}{ll}8 & (2,5) \\
2 & (0,6) \\
1 & (0,3) \\
4 & (1,3) \\
1 & (0,3)\end{array}$ & 0,12 \\
\hline Riesgo de depresión & $92(29,2)$ & 0,91 \\
\hline
\end{tabular}

*Basado en Log Rank Test. Las variables categóricas, se muestran como frecuencias y porcentajes.

Tabla 4. Métodos de ayuda diagnóstica y su asociación con sobrevida en pacientes con co-infección VIH-TBC. Lima, Perú. 2004-2012

\begin{tabular}{|c|c|c|}
\hline Variables & Total $(n=315)$ & Valor $p^{*}$ \\
\hline \multicolumn{2}{|l|}{ Radiografía de tórax } & \multirow[t]{7}{*}{0,17} \\
\hline Normal & $119(37,8)$ & \\
\hline Infiltrado apical & $9(2,9)$ & \\
\hline Infiltrado cavitario & $12(3,8)$ & \\
\hline Infiltrado miliar & $4(1,3)$ & \\
\hline Otro infiltrado & $72(22,9)$ & \\
\hline Informe no disponible & $99(31,4)$ & \\
\hline \multirow{3}{*}{$\begin{array}{l}\text { Anemia } \\
\text { Mujeres } \\
\text { Varones }\end{array}$} & $69(21,9)$ & \multirow[t]{3}{*}{0,25} \\
\hline & $51(16,2)$ & \\
\hline & $18(5,7)$ & \\
\hline \multirow{2}{*}{$\begin{array}{l}\text { Alanina aminotransferasa } \\
\text { Normal ( } \leq 40 \mathrm{U} / \mathrm{L})\end{array}$} & $97(30,8)$ & \multirow[t]{2}{*}{0,75} \\
\hline & $218(69,2)$ & \\
\hline \multicolumn{2}{|c|}{ Carga viral al diagnóstico de TBC (copias ARN/ml) } & \multirow[t]{2}{*}{0,81} \\
\hline$<11,74 \log _{10}$ & $124(50)$ & \\
\hline \multicolumn{2}{|l|}{ LT CD4 al diagnóstico de TBC } & \multirow[t]{2}{*}{0,59} \\
\hline$<200$ céls $/ \mathrm{mm}^{3}$ & $195(61,9)$ & \\
\hline
\end{tabular}

*Basado en Log Rank Test. Las variables categóricas, se muestran como frecuencias y porcentajes. TBC: tuberculosis. 
Tabla 5. Asociación ajustada entre variables y sobrevida pacientes con co-infección VIH-TBC. Lima, Perú. 2004-2012. Resultados del análisis multivariado (Método de riesgos proporcionales de Cox)

\begin{tabular}{|c|c|c|c|c|c|c|}
\hline \multirow[b]{2}{*}{ Variable } & \multicolumn{6}{|c|}{ Análisis multivariado } \\
\hline & Hazard Ratio & IC [95\%] & Valor de $p$ & Hazard ratio & IC [95\%] & Valor de $p$ \\
\hline Edad $>34$ años & 1,80 & {$[1,15-2,82]$} & $<0,01$ & 1,76 & {$[1,12-2,74]$} & 0,01 \\
\hline Peso & 0,96 & {$[0,94-0,98]$} & $<0,01$ & 0,96 & {$[0,94-0,98]$} & $<0,01$ \\
\hline Linfadenopatía & 1,38 & {$[0,79-2,41]$} & 0,24 & - & - & - \\
\hline Hospitalización & 1,63 & {$[0,98-2,71]$} & 0,05 & 1,69 & {$[1,02-2,80]$} & 0,04 \\
\hline Esquema de TARGA & 0,33 & {$[0,20-0,52]$} & $<0,01$ & 0,31 & {$[0,20-0,50]$} & $<0,01$ \\
\hline Esquema de tuberculosis & 1,42 & {$[0,80-2,54]$} & 0,22 & - & - & - \\
\hline Patología infecciosa diferente a tuberculosis & 1,74 & {$[1,09-2,80]$} & 0,02 & 1,88 & {$[1,19-2,98]$} & $<0,01$ \\
\hline
\end{tabular}

TARGA: terapia anti-retroviral de gran actividad. Esquema de tuberculosis: tratamiento para tuberculosis acorde a la sensibilidad a fármacos del núcleo de tratamiento (rifampicina/isoniazida).

de un hospital nacional de Lima-Perú se observó que hasta $32,2 \%$ de los pacientes con infección por VIH, presentaron co-infección con TBC; lo que sugiere, que los pacientes infectados por VIH desarrollarán TBC en algún momento de sus vidas ${ }^{23}$. Estudios señalan que la TBC fue la primera causa de hospitalización y muerte al inicio de la terapia TARGA en pacientes co-infectados con VIH-TBC ${ }^{14}$. De igual manera, esta investigación presenta conclusiones similares, informando que los pacientes hospitalizados al momento del diagnóstico de la co-infección tienen $69 \%$ más riesgo de fallecer.

Presentar una patología distinta a TBC ha mostrado afectar el pronóstico de los pacientes co-infectados. Según Agbor y cols., padecer de enfermedades definitorias de estadio SIDA y no SIDA, estaría asociado a menor sobrevida $^{20}$. De igual forma, Ismail y cols., encuentran que tener una infección diferente a TBC se asocia a mayor riesgo de muerte (HR: 3,61) ${ }^{19}$. En la población de estudio, de un total de 83 pacientes fallecidos, $37,3 \%$ padecieron de alguna patología diferente a la co-infección. Dicho resultado se ve reflejado en el análisis multivariado, el que demuestra que tener como antecedente una patología distinta a TBC se asocia a $88 \%$ más riesgo de mortalidad. Así mismo, Dean y cols., resaltan que encontrarse inmunosuprimido predispone a contraer diferentes co-morbilidades, y por lo tanto, se asocia a mayor riesgo de mortalidad ${ }^{24}$.

Antes de la instauración del tratamiento anti-retroviral, la prevalencia de co-infección VIH-TBC se presentaba de manera frecuente. Estudios internacionales reportaron cifras de 33\% de pacientes co-infectados durante los años 1993-199425. En el Perú, Lama reportó hallazgos similares durante los años 1986-1993 (co-infección VIH-TBC de $33,8 \%)^{16}$.

Para el año 2008, se estimó una tasa de 1,4 millones de casos de TBC en pacientes con infección por VIH en el mundo ${ }^{26}$, siendo considerada la infección oportunista inicial en $28 \%$ de las personas con infección por VIH y hasta en 50\% en estadio SIDA. La mortalidad secundaria a la co-infección ha disminuido considerablemente desde el inicio de la administración de TARGA ${ }^{27}$, observándose estos cambios en Perú desde mayo del 2004․ Estudios reportan que, en áreas endémicas de TBC, el uso de TARGA está asociado a una significante reducción de la tasa de incidencia de adquirir TBC, disminuyendo de $31,7 / 100$ a 11,4/100 por año ${ }^{25}$. En Perú, el TARGA se fundamenta en tres fármacos base: efavirenz, nevirapina o inhibidor de la proteasa (lopinavir, atazanavir o saquinavir) reforzados con ritonavir, sumado a dos inhibidores nucleósidos de la transcriptasa reversa. En el hospital de referencia, se emplea efavirenz como fármaco base para el tratamiento anti-retroviral en personas co-infectadas con TBC, decisión abalada por recomendaciones de la OMS, las que indican que se debe iniciar el tratamiento TARGA tan pronto como sea posible y dentro de las ocho semanas de iniciado el tratamiento anti-TBC, a fin de minimizar la presencia de efectos adversos tóxicos ${ }^{9,28}$. Además, establece que efavirenz debe ser el fármaco base en el tratamiento anti-retroviral que se administre en estos casos. Al igual que en países sudafricanos, efavirenz es utilizado como núcleo de tratamiento anti-retroviral debido a su baja interacción con fármacos anti-TBC. En esta investigación se encontró que los pacientes con co-infección VIH-TBC que reciben TARGA tienen 69\% de menos riesgo de mortalidad en comparación a los pacientes que no reciben tratamiento anti-retroviral, lo que refuerza lo expuesto anteriormente.

Como se sabe, existe una delgada línea entre la TBC y la malnutrición, estableciéndose un círculo vicioso entre 
ambas. A su vez, el riesgo de adquirir TBC es mayor en individuos inmunocomprometidos, lo que explica el aumento en la prevalencia de la misma en pacientes con infección por VIH. Las alteraciones nutricionales en pacientes con $\mathrm{TBC}$, infección por VIH o co-infección, son variadas y la demanda energética conlleva a diversos estados nutricionales, los cuales pueden verse reflejados en el peso del paciente. Además, se dice que la pérdida de peso $>10 \%$ está asociada a menor sobrevida; por este motivo, se recomienda evaluar el peso de forma rutinaria en cada evaluación médica ${ }^{25}$. En este estudio se encontró que la variable peso es un factor protector, concluyendo que por cada $\mathrm{kg}$ más de peso, el paciente presenta $4 \%$ menos riesgo de morir, lo que refuerza la idea de que la ganancia ponderal está relacionada con sobrevida.

Finalmente, si bien la introducción de la terapia anti-retroviral a mediados de los años 90 ha reducido de manera importante y a nivel mundial, la morbilidad y mortalidad relacionadas con la infección por $\mathrm{VIH}^{4,6}$, la situación en países en desarrollo como el nuestro aún no es la óptima.

Hasta la fecha, no se cuenta con publicaciones nacionales sobre el estado de salud de los pacientes con co-infección VIH-TBC. Por eso, este estudio evalúa el comportamiento de esta co-infección, con posterioridad a la introducción del TARGA en el país, con el objetivo de brindar información actualizada al médico clínico peruano a fin de mejorar la expectativa y calidad de vida de este grupo de personas.

Es importante reconocer que este estudio cuenta con ciertas limitaciones; una de ellas es que, al tratarse de un estudio retrospectivo, cabe la posibilidad de pérdidas de sujetos de estudio y registros incompletos durante el tiempo de seguimiento. Por otro lado, no se pudo incluir la variable "antecedente de TBC" ya que este dato no se encontraba consignado de forma consistente en las fichas de seguimiento en base a las cuales obtuvimos la información. Además de lo ya mencionado, cabe resaltar como limitación importante la baja tasa de diagnóstico de certeza, por medio de cultivo ( $3 \%$ en el presente estudio). Finalmente, en esta investigación no se han incluido otras variables que podrían aparecer durante el seguimiento y tener impacto en el resultado final.
En conclusión, encontramos que la edad, el encontrarse hospitalizado y la presencia de co-morbilidades se asociaron a una menor sobrevida, mientras que el TARGA y un mayor peso fueron factores asociados a un mejor pronóstico. La administración oportuna del TARGA optimizando su adherencia, el garantizar un adecuado soporte nutricional y el manejo integral incluyendo las comorbilidades existentes, deben ser elementos prioritarios en el manejo de la co-infección VIH-TBC.

Agradecimientos. A los miembros del Servicio de Infectología del Hospital Nacional Arzobispo Loayza por brindarnos facilidades para la elaboración de esta investigación, y a nuestras familias, por ser el soporte durante estos años.

\section{Resumen}

Introducción: La principal causa de muerte en pacientes infectados con VIH es la tuberculosis (TBC). Pocos estudios latinoamericanos han evaluado la sobrevida de pacientes co-infectados. Objetivo: Determinar factores asociados a sobrevida en pacientes con co-infección VIH-TBC atendidos en un hospital peruano. Materiales y Métodos: Estudio de cohorte, retrospectivo, en base a registros clínicos de pacientes atendidos en el Servicio de Infectología del Hospital Nacional Arzobispo Loayza durante los años 2004-2012. Se evaluó la sobrevida de 315 pacientes, utilizando las curvas de Kaplan-Meier y el método de Riesgos Proporcionales de Cox. Resultados: De 315 pacientes, 82 murieron durante el seguimiento. La mediana de seguimiento para cada participante fue de 730 días. El análisis multivariado mostró que recibir TARGA (HR: 0,31; IC: 0,20-0,50; $<<0,01$ ) y tener mayor peso (HR: 0,96; IC 0,94-0,98; $\mathrm{p}<0,01$ ) al momento del diagnóstico de la co-infección fueron factores protectores; mientras que tener una patología distinta a TBC (HR: 1,88; IC: $1,19-2,98 ; \mathrm{p}<0,01$ ), edad mayor a 34 años (HR: 1,76; IC: $1,12-2,74 ; p \leq 0,01)$, y estar hospitalizado al momento del diagnóstico (HR: 1,69; IC 1,02-2,80; $p<0,04$ ) se asociaron a menor sobrevida. Discusión: Recibir TARGA y tener mayor peso al momento del diagnóstico de la coinfección se asociaron a mayor sobrevida.

\section{Referencias bibliográficas}

1. VIH/SIDA. OMS: Organización Mundial de la Salud [Internet]. 2017 Jul.

Disponible en: http://www.who.int/ mediacentre/factsheets/fs360/es/ (Último acceso noviembre del 2017).

2. La epidemia mundial de sida-datos claves mundiales. Disponible en: http://www.unaids.org/es/resources/ campaigns/2014/2014gapreport/factsheet (Último acceso enero del 2015).

3. Echavarria J, López de Castilla D, Iglesias D, Seas C, Gonzáles E, Maldonado F, et al. Efecto de la terapia antirretroviral de gran actividad (TARGA) en pacientes enrolados en un hospital público en Lima-Perú. Rev Med Hered 2007; 18: 184-91.

4. Lawn SD, Harries AD, Meintjes G, Getahun H, Havlir DV, Wood R. Reducing deaths from tuberculosis in antiretroviral treatment programmes in sub- Saharan Africa. AIDS. 2012; 26: 1-23.

5. Carvalho BM, Jalles A, Pires R, Barbosa T, Cunha C. Factors related to HIV/tuberculosis coinfection in a Brazilian reference hospital. Braz J Infect Dis. 2008; 12: 281-6.

6. Karo B, Haas W, Kollan C, GunsenheimerBartmeyer B, Hamouda O, Fiebig L, et al. Tuberculosis among people living with HIV/ 
AIDS in the German ClinSurv HIV Cohort: long-term incidence and risk factors. BMC Infect Dis 2014; 14: 2-12.

7. Hablemos de Tuberculosis y VIH [Internet]. Disponible en: http://www.who.int/tb/ challenges/hiv/talking_points/es/ (Último acceso enero del 2015).

8. Boletín Epidemiológico Mensual. Disponible en: http://www.dge.gob.pe/portal/docs/ vigilancia/vih/Boletin_2016/agosto.pdf (Último acceso enero del 2017).

9. Mendoza A, Iglesias D. Tuberculosis en pacientes con VIH/SIDA. Acta Méd Peruana 2008; 25: 247-54.

10. Hablemos de Tuberculosis y VIH [Internet]. Organización Mundial de la Salud. Disponible en: http://www.who.int/tb/challenges/hiv/ talking_points/es/ (Último acceso febrero del 2015).

11. Charbonnier F, Calmy A, Janssens J. Co-infection tuberculose et VIH: enjeux thérapeutiques. Rev Med Suisse. 2011; 7: 295 300 .

12. Namme LH, Marie-Solange D, Mbatchou H, Temfack E, Joko H, Kuaban C. Extrapulmonary tuberculosis and HIV coinfection in patients treated for tuberculosis at the Douala General Hospital in Cameroon. Ann Trop Med Public Health. 2013; 6: 100-4.

13. Dierberg K, Chaisson R. E. HIV-associated tuberculosis: update on prevention and treatment. Clinics Chest Med 2013 ; 34 : 21728.

14. Reyes A, Capo de Paz V, Díaz M, Pérez J, Bouza Y. Cambios en la supervivencia de enfermos con SIDA y tuberculosis en Cuba en la era del Tratamiento Antirretroviral de Alta Eficacia (TARVAE). Invest Clin. 2008;
49: 309-20.

15. Carvalho B, Monteiro A, Pires J, Grangeiro T, Frota C. Factors related to HIV/tuberculosis coinfection in a Brazilian reference hospital. Braz J Infect Di. 2008; 12: 281-6.

16. Un paso adelante en la lucha contra el SIDA [internet]. Disponible en: http://www.dge.gob. pe/vigilancia/vih/Boletin_2013/enero.pdf. (Último acceso enero del 2017).

17. Rivero A, Federico P, Iribarren J, Caylá J, Miró J, Moreno S, et. al. Recomendaciones de GESIDA/Secretaría del Plan Nacional sobre el Sida para el tratamiento de la tuberculosis en adultos infectados por el virus de la inmunodeficiencia humana (actualización enero de 2013). Enf infec Microbiol Clin 2013; 13: 672-84.

18. Lama J. Epidemiología, clínica y pronóstico de la tuberculosis en los pacientes infectados por el virus de inmunodeficiencia humana. Universidad Peruana Cayetano Heredia. 1994.

19. Ismail I, Bulgiba A. Predictors of death during tuberculosis treatment in TB/HIV co-infected patients in Malaysia. PLoS ONE 2013; 8: $1-7$.

20. Agbor A, Bigna J, Billong S, Tejiokem M, Ekali G, Plottel C, et al. Factors associated with death during tuberculosis treatment of patients co-infected with HIV at the Yaoundé Central Hospital, Cameroon: An 8-year hospital-based retrospective cohort study (2006-2013). PLoS ONE 2014; 9: 1-22.

21. Barlett J. Tuberculosis and HIV infection: partners in human tragedy. J Infect Dis 2007; 196: $124-5$

22. Català L, Orcau A, García de Olalla $\mathrm{P}$, Millet $\mathrm{J}$, Rodríguez-Mondragón A, Caylá J, et al. Survival of a large cohort of HIV-infected tuberculosis patients in the era of highly active antiretroviral treatment. Int J Tuber 2011; 15: 263-9.

23. Chuquiyauri H, Verdonk B, Gonzáles E, Zamudio E, Echevarria J, Seas C, et al. Mobi-mortalidad de pacientes con tuberculosis hospitalizados en el Departamento de Enfermedades Infecciosas, Tropicales y Dermatológicas del Hospital Nacional Cayetano Heredia, Lima-Perú entre los años 1990 y 2000. Rev Med Hered 2016; 15: 203-10.

24. Dean G, Edwards S, Ives N, Matthews G, Fox E, Navaratne L, et al. Treatment of tuberculosis in HIV-infected persons in the era of highly active antiretroviral therapy. AIDS 2002; 16: 76-83.

25. Alvarez-Uria G, Pakam R, Midde M, Naik P K. Incidence and mortality of tuberculosis before and after initiation of antiretroviral therapy: an HIV cohort study in India. J Int AIDS Soc 2014; 17: 19251.

26. Tuberculosis and HIV. Geneva, Switzerland: WHO. Disponible en: http://www.who.int/hiv/ topics/tb/en/index.html (Último acceso enero del 2015).

27. Girardi E, Palmieri F, Cingolani A, Ammassari A, Petrosillo N, Gillini L, et al. Changing clinical presentation and survival in HIV-associated tuberculosis after highly active antiretroviral therapy. J Acquir Immune Defic Syndr 2001; 26: 326-31.

28. Resolución Ministerial del Ministerio de Salud del Perú. Norma técnica de salud de atención integral del adulto con infección por el virus de la Inmunodeficiencia Humana (VIH). $1^{\text {er }}$ ed. Lima: Ministerio de Salud del Perú; 2014, p. 1-68. 